\title{
PRECESSION AND GALACTIC ROTATION ON THE BASIS OF VARIOUS PROPER MOTION SYSTEMS
}

\author{
W. FRICKE
}

\section{Previous Results}

Previous determinations of precession on the basis of proper motions resulted in corrections to Newcomb's value of lunisolar precession in the interval +0 " $.75 \leqslant \Delta p_{1}$ $\leqslant+1^{\prime \prime} .50$ per century. From a compilation of several determinations and their reduction to the average of the systems of FK3 and N30 Morgan and Oort (1951) obtained:

$$
\Delta p_{1}=+0.75, \quad P=\frac{A}{4 \cdot 74}=+0.43, \quad Q=\frac{B}{4 \cdot 74}=-0.15
$$

per century, where $A$ and $B$ are Oort's constants. Values in the system of FK4 were derived by Bošniakovich (1965), who applied differential corrections to previous results on the basis of the systematic differences FK4-FK3.

For the following reasons it was found appropriate to investigate in detail whether, on the basis of FK4, some improvement can be made in the determination of precession and galactic rotation:

(1) The differential method of reducing results from one proper motion system to another yields reliable results only on the condition that all important characteristics of the primary solution are taken into account. In most of the previous differential determinations such characteristics of primary solutions as the distribution of the stars in the sky and the different weights assigned to proper motions in different parts of the sky were disregarded.

(2) Different results obtained on the basis of different fundamental systems and from different basic material were usually regarded as caused by the deviations of the systems from each other. Identical basic material and identical methods of treatment must be applied in order to reveal the effects of differences between the proper motion systems.

(3) Some determinations were based on proper motions of faint stars after the motions had been reduced to a fundamental system. The limiting magnitude in FK3 and FK4 is about 7.5. The reduction of proper motions of fainter stars to the fundamental system may involve systematic errors depending on the magnitude unless the motions of the faint stars were measured with respect to brighter stars by techniques which exclude magnitude errors.

Perek (ed.), Highlights of Astronomy, 306-310. (C I.A.U. 
(4) In determinations of precession and galactic rotation the errors of the fundamental proper motions cannot be regarded as negligibly small. In FK4, the averaged standard errors describing the internal accuracy are

$$
\varepsilon_{\mu_{\alpha}}= \pm 0^{\prime \prime} 16, \quad \varepsilon_{\mu_{\delta}}= \pm 0.17 \text { per century }
$$

and the averaged standard errors of the system are

$$
\varepsilon_{\mu_{\alpha}}= \pm 0^{\prime \prime} 17, \quad \varepsilon_{\mu_{\delta}}= \pm 0^{\prime \prime} 07 \text { per century }
$$

These numbers represent linear averages over the whole sky. The average values resulting from internal and systematic errors combined are

$$
\varepsilon_{\mu_{\alpha}}= \pm 0^{\prime \prime} 24, \quad \varepsilon_{\mu_{\delta}}= \pm 0.18 \text { per century }
$$

Although corresponding values for previous fundamental systems are not known, it is certain that they are not smaller. Due to these errors, and in consideration of the greater effect of the systematic errors, the weight of the component $\mu_{\alpha}$ is about one half the weight of $\mu_{\delta}$. Furthermore, it has to be taken into account that the errors are different in different parts of the sky.

(5) Williams and Vyssotsky (1947) found that the precessional corrections determined from $\mu_{\alpha}$ and $\mu_{\delta}$ of FK3 separately agree with each other, while Morgan (1952) obtained discordant results from both components in the N30 system. It appeared desirable to verify these results by carrying out solutions from $\mu_{\alpha}$ and $\mu_{\delta}$ separately on the basis of various proper-motion systems including the FK4.

Guided by the considerations outlined here two different approaches were made to determine the precessional corrections and the parameters of galactic rotation on the basis of the proper motion systems of GC, FK3, N30, and FK4.

\section{Results of a Reinvestigation of the McCormick and Cape Proper Motions of Faint Stars}

Basic material for this investigation were the McCormick and Cape proper motions of stars of average photovisual magnitude $11 \cdot 1$ presented and analysed by Williams and Vyssotsky (1947) in the FK3 system. The group averages of the motions as they were given by these authors were individually reduced to the systems of N30 and FK4. Under identical conditions, this means that with the weights and parallax factors used by Williams and Vyssotsky, solutions were carried out in the systems of FK3, N30, and FK4. It turned out that the correction to Newcomb's lunisolar precession derived from combined solutions of $\mu_{\alpha}$ and $\mu_{\delta}$ is identical in all three systems. The numerical value of the correction, however, depends sensitively on the relative weight of the McCormick and Cape proper motions. Williams and Vyssotsky had assigned to the Cape proper motions twice the weight of the McCormick motions. 
Other solutions were carried out in which equal weight was assigned to McCormick and Cape motions. Finally, one may consider solutions as well founded which are based on the McCormick motions alone, since the fundamental proper motions are less accurately known in the Southern sky than in the Northern sky, and since the reduction of the Cape motions to FK3 may be affected by serious errors. The results for $\Delta p_{1}$ are

$+10^{\prime \prime} .8 \pm 0 " 12$ from McCormick motions alone,

$+1^{\prime \prime} .38 \pm 0$ ".08 from McCormick and Cape motions with equal weight.

These results are valid for each of the systems of FK3, N30, and FK4. Since it is not possible to exclude systematic errors from the McCormick and Cape material satisfactorily, there remains the interval

$$
+1^{\prime \prime} 08 \pm 0.12 \leqslant \Delta p_{1} \leqslant+1 . " 38 \pm 0.08
$$

for the centennial value of the correction. The results of separate solutions from $\mu_{\alpha}$ and $\mu_{\delta}$ deviate from each other in all three systems FK3, N30, and FK4. In view of the noteworthy uncertainty of the $\mu_{\alpha}$-systems, this fact is not surprising.

Combined solutions from $\mu_{\alpha}$ and $\mu_{\delta}$ give the same value $Q=-0^{\prime \prime} .16 \pm 0.03$ of Oort's constant corresponding to $B=-7.6 \pm 1.4 \mathrm{~km} \mathrm{sec}^{-1} \mathrm{kpc}^{-1}$ in all systems. Oort's constant $P$ differs slightly in the three systems and depends on the weight of the proper motions in different parts of the sky. The average value of $P$ obtained from the three systems is $P=+0^{\prime \prime} .33 \pm 0^{\prime \prime} .04$ corresponding to $A=+15 \cdot 5 \pm 1.9 \mathrm{~km} \mathrm{sec}^{-1} \mathrm{kpc}^{-1}$.

All the results demonstrated that differential solutions in N30 and FK4 with respect to a primary solution in FK3 give erroneous results, if the particularities of the primary solution are disregarded. The determinations made by Morgan and Oort (1951) and by Bošniakovich (1965) were affected by such errors.

\section{Results from Fundamental Proper Motions of Distant Stars}

Basic material for this investigation were the proper motions of 512 FK4/FK4 Sup stars with distances greater than about 100 parsec. Obvious advantages of this material for the determination of precession and galactic rotation are:

(1) All proper motions are individually known in the systems GC, FK3, N30, and FK4 so that errors in the reduction from one system to the other are excluded.

(2) The motions in FK3, N30, and FK4 are presumably much less affected by errors depending on the magnitude than the motions of faint stars.

(3) For most of these stars radial velocities are known so that peculiarities of the stellar motions can be investigated and a check of the photometric distance scale made.

Solutions for the solar motion, the precessional corrections, and the parameters of galactic rotation were performed under strictly identical conditions on the basis of the proper motions directly taken from GC, FK3, N30, and FK4. Effects arising 
from anomalies in the distance distribution of the stars, from fluctuations in the velocity field due to star streams and from errors in the $\mu_{\alpha}$ and $\mu_{\delta}$ systems, were investigated by carrying out a number of alternative solutions.

The most essential result is that the correction to Newcomb's lunisolar precession has the same value, within 0".01 per century, in the systems FK3, N30, and FK4,

$$
\Delta p_{1}=+1 . " 10 \pm 0.10 \text { (p.e.) per century }
$$

The value $\Delta \lambda+\Delta e$ describing the combined correction due to incorrect planetary precession and an unprecessional motion of Newcomb's equinox is

$$
\Delta \lambda+\Delta e=+1{ }^{\prime \prime} 20 \pm 0.11 \text { per century }
$$

in both FK4 and N30, while the value is slightly different in FK3 and GC. The parameters of galactic rotation in the systems FK4 and N30 are (in units of $\mathrm{km} \mathrm{sec}^{-1} \mathrm{kpc}^{-1}$ )

$$
\begin{array}{lll}
\text { FK } 4: & A=+14 \cdot 2 \pm 1 \cdot 9, & B=-11 \cdot 8 \pm 1 \cdot 9 \\
\text { N30: } & A=+17 \cdot 1 \pm 1 \cdot 9, & B=-10 \cdot 0 \pm 1 \cdot 9 .
\end{array}
$$

GC and FK3 reveal deviations, which are not greater than may be expected from the lower accuracy of these systems.

In the material the Scorpio-Centaurus association is represented by twenty members, and some other well-known associations by a smaller number of stars. The complete exclusion of the Scorpio-Centaurus stars caused no appreciable alteration in the results.

By dividing the material into stars nearer than 250 parsec and those farther away it was found that the local group of stars shows no indication of a rotation deviating from differential galactic rotation.

\section{Recommended Values of the Precessional Corrections}

For investigations to be based on proper motions freed from the effects of incorrect precession and of the zero point error in $\mu_{\alpha}$, it is recommended that the corrections

$$
\begin{aligned}
\Delta n & =+0.44 \text { corresponding to } \Delta p_{1}=+1{ }^{\prime \prime} 10 \text { per century, } \\
\Delta \lambda+\Delta e & =+1.20 \text { per century }
\end{aligned}
$$

should be applied to motions in the systems FK4 and N30. Proper motions in the systems GC or FK3 should in all cases be reduced to the system of FK4, and then the precessional corrections applied. It is not recommended that, for investigations of stellar motions, averages be taken of proper motions in N30 and FK4. In case of doubt it is preferable to base investigations on each system, separately considered, in order to make apparent the variational width of results due to the differences between the best proper motion systems presently available. 


\section{References}

Bošniakovich, N.A. (1965) Bull. astr., 25, 119. (IAU-Symposium No. 21.)

Morgan, H. R. (1952) Astr. Pap. Wash., 13, Part III. (Introduction to N30.)

Morgan, H.R., Oort, J.H. (1951) Bull. astr. Inst. Netherl., 11, 379.

Williams, E., Vyssotsky, A. N. (1947) Astr. J., 53, 63.

\section{DISCUSSION}

W. Dieckvoss: Proper motions for more than 160000 stars of the AGK3 preliminary catalogue have been studied at Bergedorf. The resulting values for Oort's constants are almost identical with those found by Professor Fricke, but the mean errors, about $\pm 0.7 \mathrm{~km} \mathrm{sec}^{-1} \mathrm{kpc}^{-1}$, are smaller. The distances of these stars range between 100 and 1000 parsec.

G. van Herk: I cannot agree that the motions in FK3, N30 and FK4 are unaffected by errors depending on magnitude. The differences in $\mu_{\alpha}$ between the FK4 and N30 systems show a marked dependence on magnitude in some parts of the sky. For example, in the zone between declinations $-80^{\circ}$ and $-28^{\circ}$ the centennial $\Delta \mu_{\alpha}$ show a total average rate of -0 . $160 \pm 0$ ".026 (m.e.) per magnitude. As the brightest stars are unevenly distributed over the sky this must have some influence on the solutions, but I cannot tell how much. 